\title{
Mathematical Assessment of Physical and Chemical Processes from the Middle B to the Early F Type Main Sequence Stars
}

\author{
Kutluay Yüce ${ }^{1}$ and Saul J. Adelman ${ }^{2},{ }^{3}$ \\ ${ }^{1}$ Department of Astronomy and Space Sciences, Faculty of Science, University of Ankara, \\ TR-06100 Anadolu, Ankara, Turkey, \\ email: Kutluay.Yuce@ankara.edu.tr \\ ${ }^{2}$ Department of Physics, The Citadel, 171 Moultrie Street, Charleston, SC 29409, \\ United States of America, \\ email: adelmans@citadel.edu \\ ${ }^{3}$ Guest Investigator, Dominion Astrophysical Observatory, Victoria, Canada
}

\begin{abstract}
The middle B to the early F Main Sequence stars have some of the most quiet stellar atmospheres. In this part of the HR diagram we find stars with atmospheres in radiative equilibrium. They lack the convective circulations of the middle $\mathrm{F}$ and cooler stars and the massive stellar winds of hotter stars. When stars of different mass evolve off the Main Sequence in this part of the HR Diagram their evolutionary paths do not cross initially. Thus stars with the same effective temperature and surface gravity have the same luminosity and mass. By comparing their elemental abundances, we might be able to identify physical processes which cause any differences in their abundances. Here we begin with stars whose effective temperatures and surface gravities are similar, and which have been analyzed by us using spectra obtained from the Dominion Astrophysical Observatory (DAO). Improvements in our knowledge of the energy distributions of stars (for example via GAIA measurements) should lead to improved estimates of stellar effective temperatures and surface gravities.
\end{abstract}

\section{Introduction}

Preston (1974) formulated the designation chemically peculiar (CP) stars on the Upper Main Sequence (MS). Adelman et al. (2003) showed that two of his classes the MercuryManganese $(\mathrm{HgMn})$ and the metallic-line $(\mathrm{A} m$ ) stars were a single class. Evolutionary tracks indicated that the hot A $m$ stars (spectral type A0-A2) have evolved from the stars at the cool end of the HgMn class on the MS. Later Adelman \& Unsure (2007) discovered there is no clear demarcation between the surface abundances of those stars classified as normal and as A $m$ stars.

Yüce \& Adelman (2014) describe the detailed chemical composition characteristics of non-magnetic B, A, and early $\mathrm{F}$ type stars for 32 elements using graphical techniques to understand better the relationship of normal and non-magnetic CP stars. The spread of the abundance anomalies for a given element tends to be smaller among the Am stars than among the HgMn stars. Adelman et al. (2015) found the abundances of the SB2 component stars HR 104 A, B and $\theta$ Aql A follow the solar pattern while those of $\theta$ Aql $\mathrm{B}$ follow those of a weak non-magnetic CP star. We progress in our correlation study of the physical parameters and surface chemical composition of middle Main Squence band stars. The study has the potential for some interesting surprises to describe how we separate the A $m$ from the normal A stars using our DAO series analyses coauthored with Dr. Austin F. Gulliver. Now we propose to study non-magnetic normal and chemically peculiar B, A, and F stars by creating an index which measures the degree to which such 
stars have values between those of solar abundance A stars and the classical Am/Fm stars.

\section{Findings}

We investigate whether metallic line A $m$ and normal A stars with similar values of effective temperature and surface gravity have the same abundances obtained from high resolution fine analyses. The values of microturbulent velocity for the cooler Am stars $(\mathrm{T}<8500 \mathrm{~K})$ are greater than those of both the hotter Am and all normal stars. The microturbulent velocity increases from $2 \mathrm{~km} \mathrm{~s}^{-1}$ for early A $m$ type stars with $\mathrm{T} \geqslant 8500$ $\mathrm{K}$ to $5 \mathrm{~km} \mathrm{~s}^{-1}$ for the late Am stars.

For Am stars our index is based on log $g$, and systematic chemical peculiarities in iron group and heavy elements. A key parameter for the Am phenomena is the titanium abundance. There are clearly differences between light elements (MgAlSiS) and iron group elements (ScTiVCrMnFeCoNi) for the stars in the region of $\log g \geqslant 3.90$ and for the stars evolving from the MS $(\log \mathrm{g}<3.90)$. The richness relative to the Sun in the iron group elements of the MS stars is more than those of both the light elements and the evolving from MS stars. Our computations show that Ca and/or Sc are underabundant in many MS A $m$ stars. The CaSc abundances relative to the Sun have an average value of -0.6 dex for 10 A $m$ Main Squence stars whereas 12 evolved A $m$ stars show solar values within our error limits.

For Normal A stars our index is based obtained during this study is based on log g, and systematic peculiarities in only light elements with the evolution. The relative element abundances in the light (MgAlSiS), iron group ( $\mathrm{ScTiVCrMnFeCoNi),} \mathrm{heavy} \mathrm{elements}$ $(\mathrm{BaLaCeNdEu})$ are nearly solar/normal for the normal A stars with log g close to 4 while these stars in the light elements are more underabundant than the stars evolving from the MS. The CaSc abundance relative to the Sun is solar in the averaged values for the 6 Main Squence stars and 15 evolved normal stars.

\section{Conclusion}

In this investigation we notice that the effects due to surface gravity and other physical parameters on the physical properties, and chemical structure distinction. Chemical peculiarities are clearly evident in iron group and heavy elements of the MS A $m$ stars and in light elements of the evolved normal stars within our DAO series. Another particularly surprising result, which is an indicator of the A $m$ phenomenon, is that the observed abundances of all of elements with $\mathrm{Z}>22$ are overabundant for such stars.

\section{Acknowledgements}

Dr. Kutluay YÜCE thanks Ankara University. Prof. Dr. Saul J. ADELMAN thanks Dr. James E. Hesser, Director of the DAO for the observing time. SJA's contribution to this paper was supported in part by grants from The Citadel Foundation.

\section{References}

Adelman, S. J., Adelman, A. S., \& Pintado, O. I. 2003, A\&A, 397, 267

Adelman, S. J. \& Unsuree, N. 2007, BaltA, 16, 183

Adelman, S. J., Yüce, K., \& Gulliver, A. F. 2015, PASP, 127, 509

Preston, G. W. 1974, ARAA, 12, 257

Yüce, K. \& Adelman, S. J. 2014, PASP, 126, 345 